\title{
Dynamical and invariant supersymmetry in the fermion pairing problem
}

\author{
Pavel Cejnar ${ }^{1,2, *}$ and Hendrik B. Geyer ${ }^{2, \dagger}$ \\ ${ }^{1}$ Institute of Particle and Nuclear Physics, Charles University, \\ $V$ Holešovičkách 2, 18000 Prague, Czech Republic \\ ${ }^{2}$ Institute of Theoretical Physics, University of Stellenbosch, 7602 Matieland, South Africa
}

(Dated: October 24, 2018)

\begin{abstract}
We argue that fermion-boson mapping techniques represent a natural tool for studying manybody supersymmetry in fermionic systems with pairing. In particular, using the generalized Dyson mapping of a many-level fermion superalgebra with the $\mathrm{SU}(2)$ type of pairing we investigate two kinds of supersymmetry connecting excitations in the systems with even and odd particle numbers: dynamical supersymmetry, which ensures a unified classification of states for both even and odd populations, and invariant supersymmetry with actual degeneracies of states within the same supermultiplet. Dynamical supersymmetries based on the dynamical algebra of the type $\mathrm{U}(K / 2 \Omega)$ (where $K$ and $2 \Omega$ denote the number of fermion-pair and single-fermion states, respectively) naturally arise in the bosonized description of the system. Conditions for invariant supersymmetry are determined in a restricted case of bilinear supercharge operators.
\end{abstract}

PACS numbers: 21.60.Ev, 21.60.Cs, 21.60.Fw, 03.65.Fd

\section{INTRODUCTION}

Supersymmetry is commonly known as a hypothetical algebraic scheme in quantum field theory that unifies internal and space-time symmetries and predicts elementary particles of matter occurring in boson-fermion doublets [1]. As shown by Witten [2], the same scheme can be applied in nonrelativistic quantum mechanics, yielding a generalized dynamical algebra (superalgebra) which leads to an analogous doublet structure (except for the unique ground state) in the spectra of relevant quantum systems. In the simplest case, the SUSY Hamiltonian $H=a^{\dagger} a+b^{\dagger} b$ is just the anticommutator of supercharges $Q=b a^{\dagger}$ and $Q^{\dagger}=a b^{\dagger}$ that change bosonic excitations $\left(b^{\dagger}\right)$ into fermionic ones $\left(a^{\dagger}\right)$ and vice versa, so that the excited states $\left|n_{a}, n_{b}\right\rangle$ (where $n_{a}$ and $n_{b}$, respectively, are numbers of fermionic and bosonic quanta) exhibit the characteristic two-fold degeneracy $H\left|0, n_{b}\right\rangle=H\left|1, n_{b}-1\right\rangle$, coupling boson-like and fermion-like excitations.

Applications of SUSY quantum mechanics soon followed. Analytically solvable and isospectral sets of potentials were constructed on the basis of SUSY (see Refs. [3, 4] and references therein) and some of these potentials were found relevant for experimental spectroscopic data of certain atoms and ions. Thus an (approximate) manifestation of phenomenological supersymmetry was established in atomic physics [5, 6]. Methods based on the SUSY formalism were also developed in random matrix theory and applied to systems that exhibit signatures of quantum chaos [7].

In nuclear physics, the concept of supersymmetry found a natural application (see e.g. Refs. $[8,9,10,11]$ ) in the framework of the Interacting Boson-Fermion Model [12]. The embedding of the IBFM dynamical algebra of the type $\mathrm{U}_{\mathrm{B}}(K) \otimes \mathrm{U}_{\mathrm{F}}(2 \Omega)$ (formed by $K^{2}$ bosonic generators $b_{i}^{\dagger} b_{j}$ and by $(2 \Omega)^{2}$ fermionic generators $a_{k}^{\dagger} a_{l}$, where $i, j=1, \ldots K$ and $k, l=1, \ldots 2 \Omega$ enumerate single-boson and -fermion states, respectively) into the dynamical superalgebra $\mathrm{U}(K / 2 \Omega)$ (with the mixed generators $b_{i}^{\dagger} a_{l}$ and $a_{k}^{\dagger} b_{j}$ added) makes it possible to simultaneously describe low-lying spectra of doublets $[8,10]$ or quartets [11] of neighboring even and odd nuclei using a single energy formula with SUSY-based quantum numbers. (Note that in this paper direct products are used also for algebras, although rigorously we should speak about a direct sum of generators associated with the corresponding product of groups.)

Dynamical supersymmetries are associated with chains $\mathcal{D} \supset \mathcal{A}_{1} \supset \mathcal{A}_{2} \supset \ldots$ of possible algebraic decompositions of the dynamical superalgebra $\mathcal{D}$ and refer to situations when the Hamiltonian $H$ can be written solely in terms of the Casimir invariants $C\left(\mathcal{A}_{i}\right)$ of (super)algebras in one particular chain, thus implying complete integrability with a multitude of conservation laws $\left[H, C\left(\mathcal{A}_{i}\right)\right]=0$.

It should be stressed that nuclear supersymmetry, introduced by Iachello [8] already in 1980, was historically the first application of the SUSY ideas in nonrelativistic physics. Their most detailed verification in nature up to datein the recent experimental work by Metz, Jolie et al. [13] — opened up further questions in the SUSY many-body

*Electronic address: pavel.cejnar@mff.cuni.cz

${ }^{\dagger}$ Electronic address: hbg@sun.ac.za 
physics. Unfortunately, discussions of dynamical supersymmetry on the phenomenological nuclear-structure level, and its relation to the notion of SUSY quantum mechanics, have not always clarified the distinction between them, nor focused on the microscopic basis of nuclear SUSY. It is therefore not totally surprising to find that somewhat negative opinions such as the following one by 't Hooft [14] have been voiced: "At first sight, the fact that supersymmetric patterns were discovered in nuclear physics has little to do with the question of supersymmetry among elementary particles, but it may indicate that, as the spectrum of particles is getting more and more complex, some supersymmetric patterns might easily arise, even if there is no 'fundamental' reason for their existence."

The dynamical supersymmetry described theoretically in Ref. [11], and found in experimental data [13], is based on an immediate decomposition

$$
\mathrm{U}(K / 2 \Omega) \supset \mathrm{U}_{\mathrm{B}}(K) \otimes \mathrm{U}_{\mathrm{F}}(2 \Omega) \supset \ldots
$$

of the dynamical superalgebra into a product of the corresponding bosonic and fermionic algebras, namely on the decomposition $\mathrm{U}_{\nu}(6 / 12) \otimes \mathrm{U}_{\pi}(6 / 4) \supset \mathrm{U}_{\mathrm{B} \nu}(6) \otimes \mathrm{U}_{\mathrm{B} \pi}(6) \otimes \mathrm{U}_{\mathrm{F} \nu}(12) \otimes \mathrm{U}_{\mathrm{F} \pi}(4) \supset \ldots$ in the observed case, where $\nu$ and $\pi$, respectively, stand for neutron and proton realizations of the above (super)algebras. This implies that the achieved SUSY description does not really go beyond an application of the same IBFM Hamiltonian (with fixed interaction strengths) to a quartet of neighbouring even-even, even-odd, odd-even, and odd-odd nuclei. Indeed, since the dynamical-superalgebra irreps with more than one fermion in a given nucleus are highly excited, and thus do not mix with the low-energy spectrum, the SUSY Casimir invariants can be skipped as they only yield the same additive constants to level energies in all four nuclei considered [11,13]. The remaining Casimir invariants (of the embedded algebras) are precisely those that appear in the standard IBFM description.

As recently demonstrated in Ref. [15], any collective superalgebra of fermion-pair and single-fermion operators, linked to a microscopic perspective of the IBFM, can be naturally embedded into the phenomenological-type bosonfermionic dynamical superalgebra $\mathrm{U}(K / 2 \Omega)$. Here $K$ and $2 \Omega$ now denote the number of pair and single-fermion operators, respectively, contained in the fermionic collective superalgebra. Moreover, the same analysis leads to the conclusion that all microscopically relevant dynamical supersymmetries must separately conserve the numbers of bosons and fermions, so that they are always of the form (1). Dynamical supersymmetry based on the IBFM is therefore always restricted to the structure (1), and should then probably only be referred to as dynamical bosonfermion symmetry.

An interesting, though so far mostly hypothetical possibility was, nevertheless, considered by Jolos and von Brentano [16]. It is based on the requirement that supercharges $Q_{i}$, generating the odd sector of a boson-fermion superalgebra $\mathcal{I} \subset \mathrm{U}(K / 2 \Omega)$, together with generators of the even sector, commute with the Hamiltonian, and thus $\mathcal{I}$ forms a superalgebra of invariant supersymmetry of the system. This should be satisfied regardless of whether or not the system possesses any dynamical supersymmetry of the form (1). Clearly, the invariant supersymmetry represents principally the same kind of symmetry as the one introduced by the SUSY quantum mechanics, although the form of the Hamiltonian can be more general than simply the anticommutator of supercharges discussed above. It should be stressed that the bosons considered in this work are introduced on a purely phenomenological level.

The distinction between the above two SUSY schemes is similar to the difference between invariant and dynamical symmetries in standard quantum mechanics: in the former case, all generators of the given symmetry algebra commute with the Hamiltonian, while in the latter this is satisfied only for Casimir operators of a certain chain of subalgebras (the last algebra in the chain thus represents an invariant supersymmetry). Dynamical supersymmetry, as presented in nuclear data, implies that all states in the neighbouring even and odd systems are labelled by the same set of SUSY quantum numbers, but these states are not necessarily degenerate. The invariant supersymmetry, on the other hand, results in actual degeneracy (at least on a relative scale) of a certain subset of states, which has not been observed yet. Needless to say, that in field theory, as well, the SUSY scenario naturally assumes breaking of the supersymmetry between elementary bosons and fermions, as large mass differences for particles within the same supermultiplet must be accommodated.

In this work, we extend the approach of Ref.[15] to study microscopic conditions for supersymmetric schemes in fermionic many-body systems. Our present discussion aims to clarify that while dynamical boson-fermion symmetry seems to be firmly established, and in the final instance based on the nuclear interaction in situations where some collective pairs are favoured (together with the utility of boson-fermion mappings, see Sec. II), the direct analogue in many-body systems of SUSY quantum mechanics (invariant supersymmetry), still requires clarification and may indeed be difficult to realise. This is explicitly demonstrated in Sec. III. Again the precise nature of the interaction on the fermionic level will of course turn out to be crucial for the appearance of invariant SUSY. 


\section{BOSONIZED FORMS OF THE PAIRING SYSTEM}

\section{A. Pairing Hamiltonian}

In this section we present a bosonized form of a many-level fermionic system with the SU(2) type of pairing, a standard interaction to capture the essence of superconductivity in solids and nuclei [17]. We will deal with a set of single-particle states, enumerated by $k=1, \ldots \Omega$, and the respective time-reversal conjugate states, denoted by $\bar{k}=\overline{1}, \ldots \bar{\Omega}$, occupied by an arbitrary number $N_{\mathrm{F}} \in[0,2 \Omega]$ of fermions. The fermion creation and annihilation operators corresponding to individual states are $a_{k}^{\dagger}, a_{\bar{k}}^{\dagger}$ and $a_{k}, a_{\bar{k}}$. The Hamiltonian

$$
\begin{aligned}
H_{\mathrm{F}} & =\sum_{k} E_{k}\left(a_{k}^{\dagger} a_{k}+a_{\bar{k}}^{\dagger} a_{\bar{k}}\right)-\sum_{k, l} G_{k l} a_{\bar{k}}^{\dagger} a_{k}^{\dagger} a_{l} a_{\bar{l}} \\
& =\sum_{k} E_{k} N_{k}-\sum_{k, l} G_{k l} B_{k}^{\dagger} B_{l}
\end{aligned}
$$

contains as free parameters single-particle energies $E_{k}$ (states $k$ and $\bar{k}$ are degenerate due to the time-reversal invariance) and strengths of the pairing interaction $G_{k l}=G_{l k}^{*}$. We can anticipate that the pairing interaction acts only between fermions in a certain subset $\mathcal{P} \equiv\left\{k_{1}, k_{2}, \ldots k_{\Xi}\right\}$ (with all $k_{i}$ 's mutually different, $\Xi \leq \Omega$ ) of states-most likely states in some interval around the Fermi energy - so that $G_{k l} \neq 0$ only for $k, l \in \mathcal{P}$.

The operators

$$
B_{k}^{\dagger}=a_{\bar{k}}^{\dagger} a_{k}^{\dagger}
$$

and $B_{k} \equiv\left(B_{k}^{\dagger}\right)^{+}=a_{k} a_{\bar{k}}$ create and annihilate, respectively, a pair of fermions (bifermion) on the shell $k$ (thus there are $\Omega$ such pairs), and together with the $k$-shell fermion number operator $N_{k}=a_{k}^{\dagger} a_{k}+a_{\bar{k}}^{\dagger} a_{\bar{k}}$ form the $\mathrm{SU}(2)$ algebra associated with each shell; $\left[B_{k}^{\dagger}, B_{k}\right]=N_{k}-1$. Thus the Hamiltonian (2) leads to the dynamical algebra $\bigotimes_{k} \mathrm{SU}(2)_{k}$.

For special choices of $E_{k}$ and $G_{k l}$, the Hamiltonian can be rewritten via a smaller number $(K<\Omega)$ of pairs,

$$
C_{\mu}^{\dagger}=\sum_{k} \chi_{\mu k} a_{\frac{k}{\dagger}}^{\dagger} a_{k}^{\dagger}
$$

and $C_{\mu} \equiv\left(C_{\mu}^{\dagger}\right)^{+}(\mu=1, \ldots, K)$, with the coefficients $\chi_{\mu k}$ satisfying the normalization constraint $\sum_{k} \chi_{\mu k} \chi_{\nu k}^{*}=\delta_{\mu \nu}$. The condition for the Hamiltonian reads as $H_{\mathrm{F}}=H_{\mathrm{F}}^{0}$, where

$$
\begin{aligned}
H_{\mathrm{F}}^{0} & =\text { const }+\sum_{\mu, \nu} U_{\mu \nu}\left[C_{\mu}^{\dagger}, C_{\nu}\right]+\sum_{\mu, \nu} V_{\mu \nu} C_{\mu}^{\dagger} C_{\nu} \\
& =\text { const }^{\prime}+\sum_{k}\left(\sum_{\mu, \nu} U_{\mu \nu} \chi_{\mu k} \chi_{\nu k}^{*}\right) N_{k}+\sum_{k, l}\left(\sum_{\mu, \nu} V_{\mu \nu} \chi_{\mu k} \chi_{\nu l}^{*}\right) B_{k}^{\dagger} B_{l}
\end{aligned}
$$

$\left(U_{\mu \nu}=U_{\nu \mu}^{*}, V_{\mu \nu}=V_{\nu \mu}^{*}\right.$ are arbitrary interaction constants). Since creation and annihilation operators of any set of fermion pairs, together with their commutators, closes under commutation, the dynamical algebra generating the Hamiltonian in Eq. (5) can be identified with the algebra associated with the pairs (4) instead of those in Eq. (3). We call the $C$-bifermions in Eq. (4) collective. Note that they couple fermions into pairs in entangled single-particle states, in contrast to the noncollective $B$-bifermions (3) whose wave functions are separable (except, of course, unavoidable entanglement due to the antisymmetrization). Note that Eq. (4) represents the most general ansatz for the collective pairs that conserves the form of the Hamiltonian (2).

The possibility to rewrite the pairing Hamiltonian (2) via a collective algebra with a small number of $C$-bifermions is not very exceptional. In general, it requires to satisfy a set of equations for unknown coefficients $\chi_{\mu k}$ and for interaction parameters $U_{\mu \nu}, V_{\mu \nu}$, cf. Eqs. (2) and (5). Dimensionality considerations suggest that some solutions should typically exist if $K / \Omega \geq \sqrt{1+1 / \Omega+(\Xi / \Omega)^{2}}-1$, which for $\Xi \ll \Omega$ indeed leads to $K \ll \Omega$. Let us stress here the importance of the requirement that also single-particle terms in $H_{\mathrm{F}}$, not only the interactions, are expressed via the collective algebra, see Eq. (5). For instance, the constancy of interaction strengths in Eq. (2), $G_{k l}=G$, would not imply that the simple $\mathrm{SU}(2)$ collective algebra generated by the single bifermion $C^{\dagger}=\sum_{k \in \mathcal{P}} a_{k}^{\dagger} a_{\bar{k}}^{\dagger} / \sqrt{\Xi}$, is the dynamical algebra of the problem, unless all the single-particle states subject to interactions are degenerate, i.e., $E_{k}=E$ for $k \in \mathcal{P}$ :

$$
H_{\mathrm{F}}^{0}=\sum_{k \notin \mathcal{P}} E_{k} N_{k}+E N_{\mathcal{P}}-G \Xi C^{\dagger} C
$$


where $N_{\mathcal{P}}=\sum_{k \in \mathcal{P}} N_{k}$.

Another example of the use of a collective algebra concerns a system of fermions on $K$ single- $j$ shells, $j=$ $j_{1}, j_{2}, \ldots, j_{K}$, with a Hamiltonian expressed just via interactions of $J=0$ pairs on individual shells, $S_{\mu}^{\dagger}=\left(a_{j_{\mu}}^{\dagger} a_{j_{\mu}}^{\dagger}\right)^{(0)}$ :

$$
H_{\mathrm{F}}^{0}=\sum_{\mu} E_{\mu} N_{\mu}-\sum_{\mu, \nu} V_{\mu \nu} S_{\mu}^{\dagger} S_{\nu}
$$

( $E_{\mu}$ are the $j$-shell energies, $N_{\mu}$ the corresponding occupation-number operators, and $V_{\mu \nu}$ the interaction strengths). Again, the Hamiltonian (7) is of the general form (2), with the conjugate states $k$ and $\bar{k}$ corresponding to opposite projections $+m_{\mu}$ and $-m_{\mu}$ of the angular momentum for the same level $\mu$.

\section{B. Mapping via noncollective pairs}

The dynamical algebra of the fermion pairing problem can be translated into boson language with available boson mapping techniques [18], mostly explored for even fermion systems. If both even and odd particle numbers are to be described simultaneously, the algebra of fermion pairs must be extended to take into account also the odd degrees of freedom. This can most naturally be done by adding the single-fermion creation and annihilation operators to the given set of bifermions. The resulting collection of operators then forms a superalgebra with the algebra of pair operators as a subalgebra.

As shown in Refs. [19, 20,21], partial bosonization of this extended superalgebra can be achieved by a generalized Dyson mapping which, in the case of the superalgebra based on the above noncollective pairs (3), leads to

$$
\begin{aligned}
B_{k}^{\dagger} & \mapsto b_{k}^{\dagger}\left(1-n_{k}-\mathcal{N}_{k}\right), \\
B_{k} & \mapsto b_{k}, \\
N_{k} & \mapsto 2 n_{k}+\mathcal{N}_{k}
\end{aligned}
$$

for the even sector, and

$$
\begin{aligned}
a_{k}^{\dagger} & \mapsto b_{k}^{\dagger} \alpha_{\bar{k}}+\alpha_{k}^{\dagger} \frac{1-\mathcal{N}_{k}-n_{k}}{1-\mathcal{N}_{k}}-\alpha_{k}^{\dagger} \alpha_{\bar{k}}^{\dagger} \alpha_{\bar{k}} \frac{1-\mathcal{N}_{k}-n_{k}}{\left(2-\mathcal{N}_{k}\right)\left(1-\mathcal{N}_{k}\right)} \\
a_{k} & \mapsto \alpha_{k}+\alpha_{\bar{k}}^{\dagger} b_{k} \frac{1}{1-\mathcal{N}_{k}}+\alpha_{k}^{\dagger} \alpha_{\bar{k}}^{\dagger} \alpha_{k} b_{k} \frac{1}{\left(2-\mathcal{N}_{k}\right)\left(1-\mathcal{N}_{k}\right)}
\end{aligned}
$$

for the odd sector [15, 21]. In the right-hand side images, $b_{k}^{\dagger}\left(b_{k}\right)$ creates (annihilates) a boson of the $k$ th type and $n_{k}=$ $b_{k}^{\dagger} b_{k}$, while $\alpha_{k}^{\dagger}$ (or $\alpha_{k}$ ) create (or annihilate) ideal fermions in the states $k$ (similarly for $\bar{k}$ ), and $\mathcal{N}_{k}=\alpha_{k}^{\dagger} \alpha_{k}+\alpha_{\bar{k}}^{\dagger} \alpha_{\bar{k}}$. These bosons and ideal fermions are kinematically independent, i.e. all boson operators commute with all fermion operators. The images of $a_{\bar{k}}^{\dagger}$ and $a_{\bar{k}}$ can be deduced from Eqs. (11) and (12), respectively, after the $k \leftrightarrow \bar{k}$ exchange of indices in the fermionic operators and inverting signs of the first term in Eq. (11) and of the second and third term in Eq. (12).

The term 'ideal fermion' is used to distinguish the fermion-type particles resulting from the mapping (where they appear as necessary ingredients of any superalgebraic extension of boson mapping techniques) from the 'real' fermions in the original formulation of the problem. The Bogoliubov-Valatin like structure of Eqs. (11) and (12) suggests a physical interpretation of ideal fermions as generalized quasiparticles [22]. Anticipating discussions that follow below, we already point out here that possible divergences associated with the denominators in these formulas simply do not appear in the physical subspace. Furthermore the loss of symmetry between creation and annihilation operators under Hermitian conjugation (a typical feature of the Dyson type of mappings) is accounted for in the calculation of observables within the general Dyson framework, see Refs.[15, 18].

The mapped Hamiltonian reads as follows:

$$
H_{\mathrm{B}}=\sum_{k} E_{k}\left(2 n_{k}+\mathcal{N}_{k}\right)-\sum_{k, l} G_{k l} b_{k}^{\dagger} b_{l}\left(1+\delta_{k l}-n_{k}-\mathcal{N}_{k}\right) .
$$

It is clear that this Hamiltonian conserves both the total number of bosons, $N_{\mathrm{B}}=\sum_{k} n_{k}$, and of ideal fermions, $\mathcal{N}_{\mathrm{F}}=\sum_{k} \mathcal{N}_{k}$, so that by considering a fixed total number of real fermions, $N_{\mathrm{F}}=\sum_{k} N_{k}$, we also fix the sum $2 N_{\mathrm{B}}+\mathcal{N}_{\mathrm{F}}$ to the given value $N_{\mathrm{F}}$; see Eq. (10).

The Dyson mapping of states can be trivially deduced from Eqs. (8)-(12). Four real-fermion basis states in each $k$-subspace yield the ideal boson-fermion images as follows: $\left.\left.\left.|0\rangle \mapsto \mid 0), a_{k}^{\dagger}|0\rangle \mapsto \alpha_{k}^{\dagger} \mid 0\right), a_{\bar{k}}^{\dagger}|0\rangle \mapsto \alpha_{\bar{k}}^{\dagger} \mid 0\right), B_{k}^{\dagger}|0\rangle \mapsto b_{k}^{\dagger} \mid 0\right)$; 
we denote real- and ideal-space vectors by angular and circled bras/kets, respectively, as in case of real and ideal vacua $|0\rangle$ and $\mid 0)$. All the states in the ideal Hilbert space that are not linear combinations of the images just given, are spurious. In particular, the components containing in any $k$-shell more than one ideal particle (whether bosons or fermions, or both) are nonphysical. We see, therefore, that the present model of pairing allows us to write the projector to the physical subspace in the following explicit form:

$$
P_{\mathrm{ph}}=\prod_{k}\left(P_{n_{k}=0} P_{\mathcal{N}_{k}=0}+P_{n_{k}=0} P_{\mathcal{N}_{k}=1}+P_{n_{k}=1} P_{\mathcal{N}_{k}=0}\right)
$$

where $P$ 's on the right-hand side represent projectors onto the ideal subspaces with the given number of ideal particles (bosons or fermions) of the $k$ th type.

Because of the nonunitary character of the Dyson mapping, the boson-fermionic Hamiltonian in Eq. (13) (as well as images of other physical observables) is non-Hermitian with respect to the standard boson-fermion Fock space inner product. However, it is well known that Dyson mappings lead to so-called quasi-Hermitian structures which are consistent with standard quantum mechanics, and in particular guarantees real eigenvalues for observables (see Refs.[15, 23, 24]). Although explicit hermitization has been achieved in some particular cases [24], it seems that this procedure will generally introduce higher order many-body interaction terms into the Hamiltonian. Fortunately, in the present case, it can be easily checked that the Hamiltonian in Eq. (13) is already Hermitian within the physical subspace, i.e., it satisfies $H_{\mathrm{B}} P_{\mathrm{ph}}=H_{\mathrm{B}}^{\prime} P_{\mathrm{ph}}$, where

$$
\begin{aligned}
H_{\mathrm{B}}^{\prime} & =\sum_{k} E_{k}\left(2 n_{k}+\mathcal{N}_{k}\right)-\sum_{k, l} G_{k l} b_{k}^{\dagger} b_{l} \\
& =\sum_{k} E_{k} \mathcal{N}_{k}+\sum_{k}\left(2 E_{k}-G_{k k}\right) n_{k}-\sum_{k \neq l} G_{k l} b_{k}^{\dagger} b_{l}
\end{aligned}
$$

is manifestly Hermitian. We see that the Hamiltonian (15) contains no interaction terms - it is just a combination of bosonic and fermionic mean fields - although both boson-boson and boson-fermion interactions were present in the original mapped Hamiltonian (13). This implies that the interactions in Eq. (13) do not affect physical states.

The Hamiltonian (15) has the same form as the one in Eq. (3) of Ref. [25]. Note that while in their treatment of pairing the authors of the cited work introduce boson-like particles, so-called cooperons, by modifying fundamental anticommutation relations of real fermions, our bosons result from the mapping of a conventional multifermionic superalgebra. In contrast to cooperons, the bosons in our case obey ordinary commutation relations, but for the limitations concerning the physical subspace they are in fact hard-core bosons, i.e., bosons with occupation numbers restricted to 0 and 1 .

In spite of the single-particle form of the Hamiltonian (15), its diagonalization in terms of some new bosons $d_{l}^{\dagger}=\sum_{k} \beta_{l k} b_{k}^{\dagger}$ and identification of the ground state for even systems with a condensate

$$
\text { (cond) } \left.\propto\left(\sum_{k} \eta_{k} b_{k}^{\dagger}\right)^{N_{\mathrm{B}}} \mid 0\right)
$$

(where $N_{\mathrm{B}}=N_{\mathrm{F}} / 2$ and $\eta_{k}=\beta_{l_{0} k}$, with $l_{0}$ denoting the $d$-boson with minimum energy), are not physically allowed operations. Indeed, such a procedure does not keep under control physicality of the transformed states; the condensate state has a finite spurious admixture as it contains terms with more than one boson on a given $k$-shell (cf. Ref. [26]). The overlap of the state (16) with the physical subspace of the ideal space is given by the following expression:

$$
\frac{\mid\left.\left(\text { cond }\left|P_{\mathrm{ph}}\right| \text { cond }\right)\right|^{2}}{(\text { cond } \mid \text { cond })}=\frac{\left|\sum_{k_{1} \neq k_{2} \neq \ldots \neq k_{N_{\mathrm{B}}}} \eta_{k_{1}} \eta_{k_{2}} \ldots \eta_{k_{N_{\mathrm{B}}}}\right|^{2}}{\left(\sum_{k}\left|\eta_{k}\right|^{2}\right)^{N_{\mathrm{B}}} N_{\mathrm{B}} !}
$$

which is less than unity for $1<N_{\mathrm{B}} \leq N_{\mathrm{F}} / 2$ and any nontrivial set of $\eta$ 's. At the same time, $P_{\mathrm{ph}} \mid$ cond) is generally not an eigenstate of $H_{\mathrm{B}}^{\prime}$, nor of $H_{\mathrm{B}}$.

On the other hand, properties of the ground state of an even system can be estimated from the bosonic equivalent of the BCS approximation [17], using the image of the BCS state $|\mathrm{BCS}\rangle=\prod_{k}\left(u_{k}+v_{k} B_{k}^{\dagger}\right)|0\rangle$, namely

$$
\left.\left.\mid \mathrm{BCS})=\prod_{k}\left(u_{k}+v_{k} b_{k}^{\dagger}\right) \mid 0\right)=P_{\mathrm{ph}} \exp \sum_{k}\left(\ln u_{k}+\frac{v_{k}}{u_{k}} b_{k}^{\dagger}\right) \mid 0\right)
$$

with the normalization condition $u_{k}^{2}+v_{k}^{2}=1$ for each $k$. The trial wave function (18) yields the following energy functional:

$$
\left(\mathrm{BCS}\left|H_{\mathrm{B}}^{\prime}-2 \lambda N_{\mathrm{B}}\right| \mathrm{BCS}\right)=\sum_{k}\left(2 E_{k}-G_{k k}-2 \lambda\right) v_{k}^{2}-\sum_{k \neq l} G_{k l} u_{k} v_{k} u_{l} v_{l}
$$


(with $\lambda$ denoting a Lagrange multiplier), the same expression as in the standard BCS approximation.

We see that the bosonic BCS state (18) is just an ordinary Glauber coherent state projected onto the physical subspace (while the original BCS state is a generalized coherent state of the fermion dynamical group [27]). If the exponential on the right-hand side in Eq. (18) is projected onto a fixed number of particles (instead of the projection onto the physical space) one would get exactly the condensate state (16) with $\eta_{k}=v_{k} / u_{k}$, but - as discussed abovethis would not be a physically justified procedure. Instead, the use of $P_{\mathrm{ph}}$ leads to the cutoff of spurious components, which also modifies the normalization factor in front of $\left.\exp \sum_{k} \eta_{k} b_{k}^{\dagger} \mid 0\right)$ in Eq. (18) with respect to the standard bosonic coherent state.

\section{Mapping via collective pairs}

If the pairing Hamiltonian can be expressed in the form (5), one can perform the fermion-boson mapping via the corresponding collective pairs. This is particularly simple in both the $\mathrm{SU}(2)$ special cases discussed above, see Eqs. (6) and (7): The $C$-bifermion from Eq. (6) yields a $c$-boson, $C^{\dagger} \mapsto c^{\dagger}[1-(n+\mathcal{N}) / \Xi]$, while the $S$-bifermions from Eq. (7) map onto s-bosons as follows: $S_{\mu}^{\dagger} \mapsto s_{\mu}^{\dagger}\left[1-\left(n_{\mu}+\mathcal{N}_{\mu}\right) /\left(j_{\mu}+\frac{1}{2}\right)\right]$. Note that the bifermion annihilation operators are in both cases mapped trivially just onto the corresponding boson annihilation operators, and the fermion number operators from the collective algebras onto analogous combinations as in Eq. (10). For instance, $N_{\mu} \mapsto 2 n_{\mu}+\mathcal{N}_{\mu}$, where $n_{\mu}$ and $\mathcal{N}_{\mu}$ stand for the boson and ideal-fermion number operators, respectively, of the $\mu$ th level in Eq. (7). It is clear that in this case the physical space is not restricted to the $s$-boson occupation numbers $n_{\mu}=0$ and 1 , but contains states with $n_{\mu}$ up to $j_{\mu}+\frac{1}{2}$. The single-fermion operators, that in both of the discussed cases supplement the bifermion algebra to yield the corresponding collective superalgebra, are also mapped analogously as in the previous section, cf. expressions in Refs. [15, 21]

The collective algebra of the bifermions (4) is not generally of the $\mathrm{SU}(2)$ type because different collective pairs, $C_{\mu}^{\dagger}$ and $C_{\nu}^{\dagger}, \mu \neq \nu$, may contain components with the same $k$. Nevertheless, using the formalism described in Refs. [20] and [15], the Dyson bosonic images can be easily constructed:

$$
\begin{aligned}
C_{\mu}^{\dagger} & \mapsto c_{\mu}^{\dagger}-\sum_{\nu} c_{\nu}^{\dagger}\left(\sum_{k} \chi_{\mu k}^{*} \chi_{\nu k} \mathcal{N}_{k}+\sum_{\omega, \pi} \sum_{k} \chi_{\mu k} \chi_{\nu k}^{*} \chi_{\omega k}^{*} \chi_{\pi k} c_{\omega}^{\dagger} c_{\pi}\right), \\
C_{\mu} & \mapsto c_{\mu} \\
{\left[C_{\mu}^{\dagger}, C_{\nu}\right] } & \mapsto-\delta_{\mu \nu}+\sum_{k} \chi_{\mu k} \chi_{\nu k}^{*} \mathcal{N}_{k}+2 \sum_{\omega, \pi} \sum_{k} \chi_{\mu k} \chi_{\nu k}^{*} \chi_{\omega k}^{*} \chi_{\pi k} c_{\omega}^{\dagger} c_{\pi} .
\end{aligned}
$$

Now $c_{\mu}^{\dagger}$ and $c_{\mu}$ create and annihilate, respectively, the boson corresponding to the $\mu$ th collective bifermion. Note, however, that single-particle creation and annihilation operators generating the odd sector of the general collective superalgebra are more difficult to determine than in the $\mathrm{SU}(2)$-based special cases [15].

It should be stressed that although the collective $C$-bifermions are just linear combinations of the noncollective ones, $C_{\mu}^{\dagger}=\sum_{k} \chi_{\mu k} B_{k}^{\dagger}$, the respective collective and noncollective bosons are not connected by the same linear relation. Note that the two kinds of boson operators can be viewed to act formally in two different Hilbert spaces, so that their comparison requires to introduce an operator $T$ which transforms the physical space of the collective mapping onto the physical space of noncollective mapping. Eq. (21) and linearity of all mapping procedures trivially yield $T c_{\mu} T^{-1} \equiv \tilde{c}_{\mu}=\sum_{k} \chi_{\mu k}^{*} b_{k}$; it means that the linear relation between $C$ - and $B$-bifermions is preserved for the respective boson annihilation operators. For the creation operators, however, the situation is much more complicated, as the application of $T$ on the appropriate linear superposition of the mapping (20) results in a difficult selfconsistent relation containing combinations of operators $\tilde{c}_{\mu}^{\dagger} \equiv T c_{\mu}^{\dagger} T^{-1}, b_{k}^{\dagger}, b_{l}$, and also $\tilde{\mathcal{N}}_{k} \equiv T \mathcal{N}_{k} T^{-1}$. This relation cannot be generally satisfied by any ansatz of the type $\tilde{c}_{\mu}^{\dagger}=\sum_{k} \chi_{\mu k}^{\prime} b_{k}^{\dagger}$, where $\chi_{\mu k}^{\prime}$ would represent some unknown coefficients, and thus the correspondence between both kinds of mapping is not linear.

\section{SUPERSYMMETRY OF THE PAIRING HAMILTONIAN}

\section{A. Possible SUSY schemes}

As explicitly demonstrated by the Hamiltonian in Eq. (15), the total number of particles is a conserved quantity in the system of bosons and ideal fermions obtained by the mapping (8)-(12). The same holds true also for any collective mapping, see Eqs. (20)-(22) (neither projection to the physical space, nor the Hermitization can spoil this property 
[15]). Therefore, the dynamics of the mapped system does not rule out the use of the $\mathrm{U}(K / 2 \Omega)$ superalgebra as the spectrum generating (dynamical) superalgebra. In fact, the superalgebra of real fermions and bifermions, either collective or noncollective, is mapped into (but generally not onto!) this superalgebra.

For the noncollective mapping, for instance, $K=\Omega$ and the even sector of $\mathrm{U}(\Omega / 2 \Omega)$ is formed by generators $b_{k}^{\dagger} b_{l}, \alpha_{k}^{\dagger} \alpha_{l}, \alpha_{k}^{\dagger} \alpha_{\bar{l}}$, and their Hermitian conjugates (all together $5 \Omega^{2}$ operators), while the odd sector is generated by $b_{k}^{\dagger} \alpha_{l}, b_{k}^{\dagger} \alpha_{\bar{l}}$, and the conjugates (all together $4 \Omega^{2}$ ) operators. We see, however, that the odd sector is not used in the construction of the Hamiltonian, so that $\mathrm{U}_{\mathrm{B}}(\Omega) \otimes \mathrm{U}_{\mathrm{F}}(2 \Omega)$ can equally well be chosen as the dynamical algebra. This means that the decomposition in Eq. (1) is applicable, which preselects possible dynamical symmetries of the system. Realizing that all these symmetries are given by standard decompositions of the $\mathrm{U}_{\mathrm{B}}(K) \otimes \mathrm{U}_{\mathrm{F}}(2 \Omega)$ algebra into chains of embedded subalgebras, we skip here their explicit discussion. In general, conditions for any specific dynamical symmetry, given by a chain $\mathcal{D} \supset \mathcal{A}_{1} \supset \mathcal{A}_{2} \supset \ldots$ of decomposition of the boson-fermion dynamical algebra $\mathcal{D}$, are obtained from the set of constraints $\left[H_{\mathrm{B}}, C\left(\mathcal{A}_{i}\right)\right]=0$ required to hold in the physical space. This naturally yields specific (for each symmetry) sets of equations for parameters $E_{k}$ and $G_{k l}$ of the Hamiltonians (15) and (2).

Although - as we just saw - the odd sector of the dynamical superalgebra $\mathrm{U}(K / 2 \Omega)$ can be ruled out, it is also clear that the SUSY quantum number $\aleph=N_{\mathrm{B}}+\mathcal{N}_{\mathrm{F}}$ still classifies all eigenstates of the mapped many-body Hamiltonian. Conservation of the total particle number is a common feature of the generalized Dyson mapping. Let us stress that this simple conclusion was not at all clear in the early days of nuclear supersymmetry, especially in view of the fact that the real fermion number maps onto the number of ideal fermions plus twice the number of bosons, $N_{\mathrm{F}} \mapsto \mathcal{N}_{\mathrm{F}}+2 N_{\mathrm{B}}$, which excludes the particle-number conservation law for real fermions from the explanation of the conservation of $\aleph$. Indeed, as shown in Ref. [15], the conservation law for $\aleph$ (as well as the separate conservation of $N_{\mathrm{B}}$ and $\mathcal{N}_{\mathrm{F}}$ ) follows from a consistent choice of the real-fermion dynamical algebra, which must represent the interaction as well as single-particle terms of the fermion Hamiltonian. Therefore, it is only the possibility of fully algebraic formulation of the fermion pairing problem that automatically creates the $\mathrm{U}(K / 2 \Omega)$-based boson-fermion description, with all potential dynamical-supersymmetry chains included.

Potential invariant supersymmetries of the pairing Hamiltonian (2) also depend on the the energies $E_{k}$ and interaction strengths $G_{k l}$. On the fermion level, all such supersymmetries are described in the framework of the most general superalgebra of multifermion operators,

$$
A_{k_{1}^{\prime}, \ldots k_{n^{\prime}}^{\prime}, \bar{k}_{1}^{\prime}, \ldots \bar{k}_{\bar{n}^{\prime}}^{\prime}}^{k_{1}, k_{n}, \bar{k}_{1}, \ldots \bar{k}_{\bar{n}}}=a_{k_{1}}^{\dagger} \ldots a_{k_{n}}^{\dagger} a_{\bar{k}_{1}}^{\dagger} \ldots a_{\bar{k}_{\bar{n}}}^{\dagger} a_{k_{1}^{\prime}} \ldots a_{k_{n^{\prime}}^{\prime}} a_{\bar{k}_{1}^{\prime}} \ldots a_{\bar{k}_{n^{\prime}}^{\prime}}
$$

where a given element belongs to the even or odd sector, respectively, according to whether the difference $\Delta N_{\mathrm{F}}=$ $(n+\bar{n})-\left(n^{\prime}+\bar{n}^{\prime}\right)$ is even or odd. Operators of the even sector transform states with even and odd fermion numbers separately, while operators of the odd sector interconnect even and odd populations. The invariant SUSY takes place if the Hamiltonian (2) commutes with any of the operators (23) belonging to the odd sector, which implies the existence of a degenerate supermultiplet of states differing by a given odd number of $\Delta N_{\mathrm{F}}$ fermions.

In the Hilbert space of bosons and ideal fermions created by the mapping (8)-(12), similarly, one can consider the superalgebra of operators

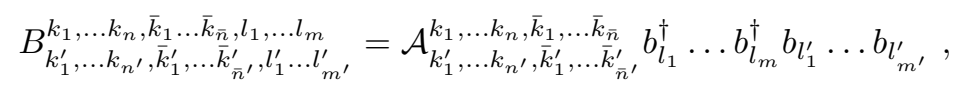

where $\mathcal{A}_{k_{1}^{\prime}, \ldots}^{k_{1}, \ldots}$ is defined in the same way as $A_{k_{1}^{\prime}, \ldots}^{k_{1}, \ldots}$ in Eq. (23), but with the real-fermion operators substituted by the corresponding ideal-fermion operators. As in the previous case, even and odd sectors of the superalgebra (24) are distinguished according to the difference $\Delta \mathcal{N}_{\mathrm{F}}$ between the numbers of creation and annihilation operators in $\mathcal{A}_{k_{1}^{\prime}}^{k_{1}, \ldots}$.

Although the mapping in Sec. II B was performed only for a small subset of the above general fermionic superalgebra, the images of operators (23) in the physical space can be constructed as the corresponding products of the images in Eqs. (11) and (12) [19]. The superalgebra (23) is thus mapped to series of operators (24), i.e., onto the given superalgebra acting in the space of bosons and ideal fermions. Trivially, even and odd sectors of the superalgebra (23) are not mixed by the mapping - the image of an element with a given value of $\Delta N_{\mathrm{F}}$ has $\Delta \mathcal{N}_{\mathrm{F}}+2 \Delta N_{\mathrm{B}}=\Delta N_{\mathrm{F}}$, where $\Delta N_{\mathrm{B}}=m-m^{\prime}$, see Eq. (24). Taking $\Delta N_{\mathrm{F}}=+1$, for example, we get an image with terms corresponding to $\left(\Delta \mathcal{N}_{\mathrm{F}}, \Delta N_{\mathrm{B}}\right)=(+1,0),(-1,+1),(+3,-1),(-3,+2), \ldots$

This expansion would hardly be tractable in its general form, but fortunately, it can be considerably simplified if states with more than one ideal fermion are physically irrelevant. Indeed, such states correspond to real-fermionic states with broken pairs which are supposed to have relatively high excitation energies. Under this restriction, the invariant-SUSY supercharges connect states in doublets with $N_{\mathrm{F}}$ and $N_{\mathrm{F}}+1$ fermions, and can be constructed just from the $\left(\Delta \mathcal{N}_{\mathrm{F}}, \Delta N_{\mathrm{B}}\right)=(+1,0)$ and $(-1,+1)$ terms. The first term applies in case that $N_{\mathrm{F}}$ corresponding to the left system in the doublet is even, the second if $N_{\mathrm{F}}$ is odd. To make contact with the standard notion of a supercharge, 
we here only focus on the second term that changes a fermion into a boson (or vice versa). It reads as

$$
\mathcal{Q}^{\dagger}=\sum_{k, l} b_{k}^{\dagger} f_{k l}\left(b_{i}^{\dagger} b_{j}, \alpha_{m}^{\dagger} \alpha_{n}\right)\left(q_{k l} \alpha_{l}+\bar{q}_{k l} \alpha_{\bar{l}}\right)
$$

where $f_{k l}$ are arbitrary functions of operators conserving separately the numbers of bosons and fermions, and $q_{k l}$ and $\bar{q}_{k l}$ are some coefficients.

In spite of the above restrictions, the commutator of the supercharge (25) with the boson-fermion Hamiltonian still remains completely unknown without a further specification of $f_{k l}$. This makes the general theoretical determination of invariant supersymmetries in fermionic systems a rather nontrivial task, where no analytic insight seems available so far. Nevertheless, the mapping procedure, as outlined in Sec. II, provides a natural framework for the classification and analysis of various special cases. In the next paragraph, for example, we determine conditions for the invariant SUSY generated by supercharges $(25)$ with $f_{k l}=1$.

\section{B. Invariant SUSY with bilinear supercharges}

We will look for conditions that the mapped Hamiltonian from Sec. II B must satisfy to commute with the simplest bilinear supercharges of the form

$$
Q^{\dagger}=\sum_{k, l} b_{k}^{\dagger}\left(q_{k l} \alpha_{l}+\bar{q}_{k l} \alpha_{\bar{l}}\right) \equiv \sum_{k, l} b_{k}^{\dagger} \hat{\alpha}_{k l}
$$

where $b_{k}^{\dagger}$ and $\alpha_{l}$ (or $\alpha_{\bar{l}}$ ) are noncollective bosons and ideal fermions, respectively, from Eqs. (8) and (12). If the supercharge is assumed to commute with the time reversal, the coefficients $q_{k l}$ and $\bar{q}_{k l}$ should be set equal (up to a phase factor), but this we do not generally require here.

The assumption $\left[H_{\mathrm{B}}^{\prime}, Q^{\dagger}\right]=0\left(=\left[Q, H_{\mathrm{B}}^{\prime}\right]^{+}\right)$leads to the following equation to be valid for any $k$ and $l$ :

$$
\left(2 E_{k}-E_{l}-G_{k k}\right) q_{k l}-\sum_{m \neq k} G_{k m} q_{m l}=0
$$

and the same also with the change of $q$ 's to $\bar{q}$ 's. For a fixed value of $l$, the condition for the existence of nontrivial solutions $q_{k l}$ and $\bar{q}_{k l}$ reads as

$$
\operatorname{det}\left(\begin{array}{cccc}
G_{11}-2 E_{1}+E_{l} & G_{12} & G_{13} & \ldots \\
G_{21} & G_{22}-2 E_{2}+E_{l} & G_{23} & \ldots \\
G_{31} & G_{32} & G_{33}-2 E_{3}+E_{l} & \ldots \\
\ldots & \ldots & \ldots & \ldots
\end{array}\right)=0
$$

This equation has rather clear physical interpretation: it says that diagonalization of the bosonic part of the singleparticle Hamiltonian (15) gives one of the eigenvalues equal to the fermionic energy $E_{l}$. Mutual conversions of the corresponding boson and fermion thus do not change the total energy. One can apply Eq. (28) simultaneously to a certain subset of fermionic states, $l \in \mathcal{S}$, which is equivalent with taking $q_{k l}=\bar{q}_{k l}=0$ for $l \notin \mathcal{S}$. The supersymmetry then concerns only a part of the spectrum.

An important special case of the above condition is obtained if one assumes supercharges with diagonal matrices of coefficients, $q_{k l}=q_{k} \delta_{k l}$ and $\bar{q}_{k l}=\bar{q}_{k} \delta_{k l}$. Eq. (27) then leads to the following simple constraints: $G_{k l}=0$ for $k \neq l \in \mathcal{S}$ and $E_{k}=G_{k k}$ for $k \in \mathcal{S}$, the coefficients $q_{k}$ and $\bar{q}_{k}$ being arbitrary for $k \in \mathcal{S}$ and zero otherwise. We thus have $H_{\mathrm{B}}^{\prime}=H_{\mathrm{SUSY}}^{\prime}$ with

$$
H_{\mathrm{SUSY}}^{\prime}=\sum_{k \in \mathcal{S}} E_{k}\left(n_{k}+\mathcal{N}_{k}\right)+\sum_{k \notin \mathcal{S}} E_{k}\left(2 n_{k}+\mathcal{N}_{k}\right)-\sum_{k, l \notin \mathcal{S}} G_{k l} b_{k}^{\dagger} b_{l}
$$

The interpretation of this solution is the same as above, with only the difference that the bosonic part of the Hamiltonian (15) is already supposed to be diagonalized a priori, allowing for the diagonal constraint on the supercharge coefficients. By inserting the above constraints into the original Hamiltonian (2), one can verify the existence of degenerated supermultiplets with $N_{k}=1$ and 2 (for $k \in \mathcal{S}$ ) directly on the fermionic level.

The Hamiltonian (29) is trivially invariant under the set of transformations $b_{k}^{\dagger} \alpha_{k}, b_{k}^{\dagger} \alpha_{\bar{k}}, \alpha_{k}^{\dagger} \alpha_{\bar{k}}, \alpha_{k}^{\dagger} \alpha_{\bar{k}}, \alpha_{\bar{k}}^{\dagger} \alpha_{\bar{k}}, b_{k}^{\dagger} b_{k}$ (and their Hermitian conjugates) with $k \in \mathcal{S}$. These are generators of the $\mathcal{I}=\bigotimes_{k \in \mathcal{S}} \mathrm{U}_{k}(1 / 2)$ superalgebra, which thus forms the invariant-SUSY superalgebra of the problem. This is so in spite of the fact that the dynamical 
algebra of $H_{\mathrm{SUSY}}^{\prime}$ in Eq. (29) is just an ordinary algebra $\mathcal{D} \subset \mathrm{U}_{\mathrm{B}}(\Omega) \otimes \mathrm{U}_{\mathrm{F}}(2 \Omega)$ where seemingly no supersymmetry is involved. In view of the discussion above, the SUSY Hamiltonian obtained from the general solution of Eq. (28) in case of nondiagonal matrices of supercharge coefficients receives the same algebraic interpretation, but with the original bosons $b_{k}^{\dagger}$ substituted by the new ones, $d_{k}^{\dagger}$, resulting from the diagonalization of the bosonic Hamiltonian. Note that the above superalgebraic scheme represents a direct generalization of the minimal SUSY scheme [2], where the invariant-SUSY superalgebra $\operatorname{sl}(1 / 1)$ is formed only by operators $Q^{\dagger}, Q$, and $H=\left\{Q^{\dagger}, Q\right\}[3,4]$. In the present case, in particular, the relevant part of the Hamiltonian is not just a supercharge anticommutator (or a sum of such terms), but a linear combination of Casimir invariants of $\mathcal{I}$.

Let us turn, at last, to the crucial question of physicality of the above supersymmetric transformations. It was so far completely ignored in this section but it is indeed very relevant since the supercharge operator (26) can transform some of the physical states out of the physical space given by the projector in Eq. (14). In particular, the supercharge operator acting within the physical space may produce a nonphysical state with $\left(n_{k}, \mathcal{N}_{k}\right)=(1,1)$ or $(2,0)$, this being not generally excluded - in case of nondiagonal matrices of supercharge coefficients - by the action of $\alpha_{l}$ or $\alpha_{\bar{l}}$, associated with $b_{k}^{\dagger}$ in Eq. (26). Consequently, some of the supersymmetric transformations that, as found above, constitute an invariant supersymmetry of the Hamiltonian $H_{\mathrm{B}}^{\prime}$ may turn to be nonphysical.

To examine this question, let us introduce a physical supercharge operator

$$
P_{\mathrm{ph}} Q^{\dagger} P_{\mathrm{ph}}=\tilde{Q}^{\dagger} P_{\mathrm{ph}}
$$

where

$$
\tilde{Q}^{\dagger}=\sum_{k \neq l} b_{k}^{\dagger} \hat{\alpha}_{k l} P_{n_{k}=0} P_{\mathcal{N}_{k}=0}+\sum_{k} b_{k}^{\dagger} \hat{\alpha}_{k k} P_{n_{k}=0}
$$

Similarly, we define

$$
\tilde{H}_{\mathrm{B}}=\sum_{k} E_{k} \mathcal{N}_{k}+\sum_{k}\left(2 E_{k}-G_{k k}\right) n_{k}-\sum_{k \neq l} G_{k l} b_{k}^{\dagger} b_{l} P_{n_{k}=0} P_{\mathcal{N}_{k}=0}
$$

that represents another form of the Hamiltonian (13). The condition for the commutation of the supercharge with the Hamiltonian in the physical space then reads as $\left[\tilde{H}_{\mathrm{B}}, \tilde{Q}^{\dagger}\right] P_{\mathrm{ph}}=0$, which yields the following operator equality,

$$
\begin{aligned}
& \sum_{k}\left(E_{k}-G_{k k}\right) b_{k}^{\dagger} \hat{\alpha}_{k k}+\sum_{k \neq l}\left(2 E_{k}-E_{l}-G_{k k}\right) b_{k}^{\dagger} \hat{\alpha}_{k l} P_{n_{k}=0} P_{\mathcal{N}_{k}=0} \\
- & \sum_{k \neq l} G_{k l} b_{k}^{\dagger} \hat{\alpha}_{l l} P_{n_{k}=0} P_{\mathcal{N}_{k}=0}-\sum_{k \neq l} G_{k l} b_{k}^{\dagger} \hat{\alpha}_{l k} P_{n_{l}=0} P_{\mathcal{N}_{l}=0} \\
+ & \sum_{k \neq l \neq m} G_{k l} b_{k}^{\dagger} \hat{\alpha}_{l m}\left(P_{n_{l}=1}-P_{n_{l}=0}\right) P_{n_{k}=0} P_{\mathcal{N}_{l}=0} P_{\mathcal{N}_{k}=0} \\
- & \sum_{k \neq l \neq m} G_{k l} b_{k}^{\dagger} b_{m}^{\dagger} b_{l} \hat{\alpha}_{m k} P_{n_{m}=0} P_{\mathcal{N}_{m}=0}=0
\end{aligned}
$$

to be valid in the physical space. It is not difficult to see that Eq. (33) can only be satisfied by imposing the above SUSY conditions for supercharges with vanishing nondiagonal $q_{k l}$ and $\bar{q}_{k l}$ elements, see Eq. (29) and the text above. The Hamiltonian (29) is the only invariant-SUSY Hamiltonian with supermultiplet states contained entirely in the physical space that can be constructed through a bilinear supercharge in Eq. (26). The other solutions, see Eq. (28), are spurious, i.e., have no real counterpart on the level of the original fermion system.

Let us finally note that the search for invariant supersymmetries would be much more involved for the mapping performed via collective bifermions from Eq. (4). In this case, we again have to check if the expression $\left[\tilde{H}_{\mathrm{B}}, \tilde{Q}^{\dagger}\right] P_{\mathrm{ph}}^{\prime}$ vanishes, where $Q^{\dagger}=\sum_{\mu, k} c_{\mu}^{\dagger}\left(q_{\mu k} \alpha_{k}+\bar{q}_{\mu k} \alpha_{\bar{k}}\right)$ is a collective bilinear supercharge and $P_{\mathrm{ph}}^{\prime}$ a projector onto the physical subspace obtained via the collective superalgebra. Unlike the noncollective case, boson-boson and bosonfermion interactions in the Hamiltonian $\tilde{H}_{\mathrm{B}}$ are generally relevant also in the physical subspace [15] and must be considered in the commutator. Moreover, the calculation is obscured by the fact that $P_{\mathrm{ph}}^{\prime}$ has a more complicated form in the general case than for the noncollective superalgebra. The above-discussed relation between collective and noncollective bosons, see the end of Sec. II C, indicates that the use of collective supercharges has a similar effect as the generalization of Eq. (26) to the form (25). 


\section{CONCLUSIONS}

We have pointed out the need to distinguish between the well-established notion of dynamical supersymmetry in many-body physics (nuclear structure in particular), and invariant supersymmetry in many-body physics as a direct analogue of SUSY quantum mechanics.

In contrast to phenomenological studies based on the IBFM, our search for the SUSY patterns is derived from purely fermionic level. Existing boson-fermion mapping techniques allow us to introduce the relevant boson and fermion degrees consistently, and facilitates our exploration of invariant supersymmetry in the simple case of pairinglike Hamiltonians.

For these Hamiltonians, and with supercharges restricted to a bilinear form, it turns out that invariant supersymmetry is ruled out for pairing other than the simplest diagonal form, see Eq. (29). This is not in contradiction with the result of Ref.[16] where the boson interaction does not follow from a mapped fermion interaction.

From a microscopic point of view, considering and accounting for the physical subspace, has been crucial for a complete analysis, as shown in detail in subsections II B and III B.

Although we have not exhausted all possibilities that may result in invariant supersymmetry for generalized pairing in a fermion system, going beyond the present analysis seems to present a tough problem when pursued analytically.

Finally, as argued in greater detail in Ref.[28], and as is also clear from the present discussion, there is no reason to expect (as, e.g., in Ref.[29]) that transfer operators in the context of dynamical supersymmetry, should be restricted to the simplest supercharges appearing in invariant supersymmetry situations. In fact, for dynamical symmetry the appropriate transfer operators are mapped images of single fermion operators, as, e.g., in Eqs. (11) and (12). In our presentation these images may indeed be considered generalized supercharges, but in general they are not of the simple bilinear type.

\section{Acknowledgments}

P.C. thanks J. Dobeš, J. Hošek, and D. Nosek for interesting discussions. This work was supported by the S.A. National Research Foundation under Grant Nos. GUN 2047181 and GUN 2044653 and by the Grant Agency of Czech Republic under Grant No. 202/02/0939.

[1] S. Weinberg, The Quantum Theory of Fields, Vol. III, Supersymmetry (Cambridge University Press, Cambridge, UK, 2000).

[2] E. Witten, Nucl. Phys. B188, 513 (1981).

[3] F. Cooper, A. Khare, and U. Sukhatme, Phys. Rep. 251, 267 (1995).

[4] F. Cooper, A. Khare, and U. Sukhatme, Supersymmetry in Quantum Mechanics (World Scientific, Singapore, 2001).

[5] V.A. Kostelecký and M.M. Nieto, Phys. Rev. Lett. 53, 2285 (1984); Phys. Rev. A 32, 1293 (1985).

[6] V.A. Kostelecký, M.M. Nieto, and D.R. Traux, Phys. Rev. D 32, 2627 (1985).

[7] H.-J. Stöckmann, Quantum Chaos. An Introduction, (Cambridge University Press, Cambridge, UK, 1999).

[8] F. Iachello, Phys. Rev. Lett. 44, 772 (1980).

[9] A.B. Balantekin, I. Bars, and F. Iachello, Nucl. Phys. A370, 284 (1981).

[10] A.B. Balantekin, I. Bars, R. Bijker, and F. Iachello, Phys. Rev. C 27, 1761 (1983).

[11] P. Van Isacker, J. Jolie, K. Heyde, and A. Frank, Phys. Rev. Lett. 54, 653 (1985).

[12] F. Iachello and P. Van Isacker, The Interacting Boson-Fermion Model (Cambridge University Press, Cambridge, UK, 1991).

[13] A. Metz, J. Jolie, G. Graw, R. Hertenberger, J. Gröger, C. Günther, N. Warr, and Y. Eisermann, Phys. Rev. Lett. 83, 1542 (1999).

[14] G. 't Hooft, The Holographic Principle, Opening Lecture at the 1999 Erice-Chalonge School, hep-th/003004.

[15] P. Cejnar and H.B. Geyer, Phys. Rev. C 65, 044313 (2002).

[16] R.V. Jolos and P. von Brentano, Phys. Rev. 60, 064318 (1999); 62, 034310 (2000); 63, 024304 (2001).

[17] J. Bardeen, L.N. Cooper, and J.R. Schrieffer, Phys. Rev. 106, 162 (1957); 108, 1175 (1957).

[18] A. Klein and E.R. Marshalek, Rev. Mod. Phys. 63, 375 (1991).

[19] J. Dobaczewski, F.G. Scholtz, and H.B. Geyer, Phys. Rev. C 48, 2313 (1993).

[20] P. Navrátil, H.B. Geyer, and J. Dobaczewski, Phys. Rev. C 52, 1394 (1995).

[21] P. Navrátil, H.B. Geyer, and J. Dobaczewski, Nucl. Phys. A 607, 23 (1996).

[22] T. Suzuki and K. Matsuyanagi, Prog. Theor. Phys. 56, 1156 (1976).

[23] F.G. Scholtz, H.B. Geyer, and F.J.W. Hahne, Ann. Phys (NY) 213, 74 (1992).

[24] G.K. Kim and C.M. Vincent, Phys. Rev. C 35, 1517 (1987). 
[25] E. Celeghini and M. Rasetti, Phys. Lett. 283A, 382 (2001).

[26] J. Dobeš, Phys. Lett. 222B, 315 (1989).

[27] W.-M. Zhang, D.H. Feng, and R. Gilmore, Rev. Mod. Phys. 62, 867 (1990).

[28] H.B. Geyer and P. Cejnar, in Proceedings of the Eleventh International Symposium on Capture Gamma-Ray Spectroscopy and Related Topics, ed. J. Kvasil, P. Cejnar, and M. Krtička (World Scientific, Singapore, 2003), p. 43.

[29] J. Barea, R. Bijker, A. Frank, and G. Loyola, Phys. Rev. C 64, 064313 (2001). 